\title{
Formulating an Odd Perfect Number: An in Depth Case Study
}

\author{
Renz Chester Rosales Gumaru ${ }^{1}$, Leonida Solivas Casuco ${ }^{2}$, Hernando Lintag Bernal $\mathbf{J r}^{3}$ \\ ${ }^{1}$ Senior High School Department, Arellano University, Pasay, Philippines \\ ${ }^{2}$ Senior High School Department, Norzagaray National High School, Norzagaray, Philippines \\ ${ }^{3}$ General Education Department, Far Eastern University - NRMF, Fairview Quezon City, Philippines
}

Email address:

renzchestergumarubsmath@gmail.com (R. C. R. Gumaru), leonidacasuco@gmail.com (L. S. Casuco),

bhambernal@gmail.com (H. L. B. Jr)

\section{To cite this article:}

Renz Chester Rosales Gumaru, Leonida Solivas Casuco, Hernando Lintag Bernal Jr. Formulating an Odd Perfect Number: An in Depth Case Study. Pure and Applied Mathematics Journal. Vol. 7, No. 5, 2018, pp. 63-67. doi: 10.11648/j.pamj.20180705.11

Received: September 17, 2018; Accepted: October 29, 2018; Published: November 30, 2018

\begin{abstract}
A perfect number is a positive integer that is equals to the sum of its proper divisors. No one has ever found an odd perfect number in the field of Number Theory. This paper review discussed the history and the origin of Odd Perfect Numbers. The theorems and proofs are given and stated. This paper states the necessary conditions for the existence of odd perfect numbers. In addition, several related studies such as "Odd Near-Perfect Numbers" and "Deficient-Perfect Numbers". Formulating odd perfect numbers will have a significant contribution to other Mathematics conjectures. This paper compiles all the known information about the existence of an odd perfect number It also lists and explains the necessary theorems and lemmas needed for the study. The results and conclusions shows the ff: Odd Perfect Numbers has a lower bound of $10^{1500}$, The total number of prime factors/divisors of an odd perfect number is at least 101, and $10^{8}$ is an appropriate lower bound for the largest prime factor of an odd perfect number and the second large stand third largest prime divisors must exceed 10000 and100 respectively. In summary, it found out that there is a chance for an odd perfect number to exist even if there is a very small possibility.
\end{abstract}

Keywords: Perfect Number, Odd, Theorem, Number Theory

\section{Introduction}

Perfect numbers have a rich history. A lot of mathematicians show interest in this topic. They were able to discover the first set of perfect numbers. According to Wikipedia, in an article entitled "Perfect Number", in about $300 \mathrm{BC}$, Euclid showed that if $2^{\mathrm{p}}-1$ is prime then $\left(2^{\mathrm{p}}-1\right) 2^{\mathrm{p}-1}$ is perfect. The first four perfect numbers were the only early discoveries of the Greek mathematicians. These numbers are 6, 28, 496, and 8128. [1]

Ismail ibnFallūs (1252), mentioned the next three perfect numbers which are 33,550,336, 8,589,869,056 and $137,438,691,328$. In a manuscript written between 1456 and 1461 , an unknown mathematician recorded the earliest European reference to a fifth perfect number, with $33,550,336$ being correctly identified for the first time. [2]

Pietro Cataldi (1588), also identified the sixth
$(8,589,869,056)$ and the seventh $(137,438,691,328)$ perfect numbers, and also proved that every perfect number obtained from Euclid's rule ends with a 6 or an 8 . Where $2^{\mathrm{p}}-1$ is prime where $\mathrm{p}$ is also prime, it is said to be a Mersenne prime. Mersenne primes are connected to Perfect Numbers because all Perfect Numbers has a factor in the form of $2^{\mathrm{p}}-1$. [3] Hence, every Mersenne prime will correspond to one perfect number. It was obvious to Mersenne's peers that he could not have tested all of these numbers, but they could not test them either. It was not until over 100 years later, in 1750, that Euler verified the next number on Mersenne's and Regius' lists, $2^{31}-1$, was prime. [4]

Lucas (1876), verified $2^{127}-1$ was also prime. Seven years later Pervouchine showed $2^{61}-1$ was prime, so Mersenne had missed this one. In the early 1900's Powers showed that Mersenne had also missed the primes 289-1 and 2107-1. [5] Finally, by 1947 Mersenne's range, $\mathrm{n}<258$, is completely checked and it was determined that the correct list is: $n=2,3$, 


\section{$5,7,13,17,19,31,61,89,107$, and 127. [6]}

The existence of an odd perfect number remains a big mystery in the field of number theory. The relevance of the existence of odd perfect number will have a great significant contribution to solve some unsolved to other math conjectures such as Pythagorean triples twin primes, and etc. [7]There are currently 49 Mersenne primes known to exist, and, therefore, 49 even perfect numbers have been discovered. The question is whether or not odd perfect numbers exist. There have been no discoveries of odd perfect numbers, although many people have been working on this problem for centuries. There have been many studies leading to a new necessary condition, or limitations, for an odd perfect number to exist. Thus this is the main purpose of this paper: to detail all of the necessary conditions for odd perfect numbers, in order to facilitate the further study of the subject. The largest perfect number is $2^{74207280}\left(2^{74207281}-1\right)$ [8]

\section{Method}

\subsection{Objective of the Study}

The study was designed to determine odd perfect number. This study specifically aimed to review the necessary conditions on the existence of an odd perfect number by:

a Lower Bound for Odd Perfect Numbers

b Perfect Numbers and Prime Divisors

c Largest Prime Factor

d On Odd Near-Perfect and Deficient- Perfect Numbers

\subsection{Methodology}

A review article or review paper is based on other published articles. It does not report original research. Review articles generally summarize the existing literature on a topic in an attempt to explain the current state of understanding on the topic. Review papers form valuable scientific literature as they summarize the findings of existing literature. It forms an idea about the existing knowledge. [9]

The purpose of a review paper is to succinctly review recent progress in a particular topic. Overall, the paper summarizes the current state of knowledge of the topic. It creates an understanding of the topic for the reader by discussing the findings presented in recent research papers.

A review paper is not a "term paper" or book report. It is not merely a report on some references you found. Instead, a review paper synthesizes the results from several primary literature papers to produce a coherent argument about a topic or focused description of a field. [10]

Qualitative research is a market research method that focuses on obtaining data through open-ended and conversational communication. This method is not only about "what" people think but also "why" they think so. The qualitative research method allows for an in-depth and further probing and questioning of respondents based on their responses, where the interviewer/researcher also tries to understand their motivation and feelings. [11]

Ethical protocol was observed as stipulated in the university research manual. Most authors who discuss qualitative research design address the importance of ethical considerations.

\subsection{Framework}

The forgoing theoretical concepts are illustrated in the paradigm that follows using the input - process - output scheme. Input is something from the external environment that is fed into the system. In an information system, the inputs may be raw data "captured" in some way or preexisting data, which has been provided by an external system. $v$ In either case, the data input is provided by an external entity. The transformation process is the most important element of a system. Without output, a system has no link back into its external environment. Output of an information system may be reports generated by an information system, or a picture on a computer monitor, or even data that is fed into another external system. - [12]

INPUT, shows the different concepts necessary in studying odd perfect numbers such as even perfect numbers, Mersenne primes, od-near-perfect and deficient-perfect numbers, and prime divisors.

PROCESS, which deals with the ways and methods to make a paper review. A narrative review explains the existing knowledge on a topic based on all the published research available on the topic. A systematic review searches for the answer to a particular question in the existing scientific literature on a topic. A meta-analysis compares and combines the findings of previously published studies, usually to assess the effectiveness of an intervention or mode of treatment. Comparing and relating related studies, as well as, collecting all theorems and facts about odd perfect numbers will be the processes in producing the desired output.

OUTPUT, which is the result of the outcomes of the input and process at is believed that studying and relating these concepts and theorems will lead to know the importance of an odd perfect number, and the necessary conditions for its existence.

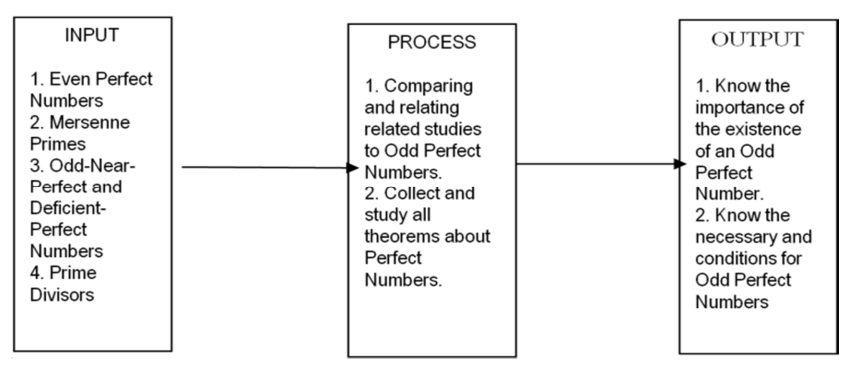

Figure 1. Research Paradigm for Existence of Odd Perfect Number.

\section{Result and Discussion}

Odd Perfect Numbers are positive integers that are odd and perfect at the same time. Odd Perfect Numbers are a positive integer that in the form $2 \mathrm{k}+1$ for $\mathrm{k}$ is any positive integer, and are equal to the sum of their proper divisors. Many mathematicians tried to prove or disprove the existence of an 
odd perfect number but so far no one has succeeded. But they were able to collect and gather the necessary conditions on the existence of an odd perfect number. They were able to classify three conditions which are the "Lower Bound for Odd Perfect Numbers", "The Number of Prime Divisors", and "The Largest Prime Factors"[13]. The following are the necessary conditions:

\subsection{Lower Bound}

One condition is the Lower Bound. This condition refers to the minimum value of an Odd Perfect Number. According to Ben Stevens on his paper entitled "A Study on Necessary Conditions for Odd Perfect Numbers".

Theorem 4.1 by Ochem and Rao: An odd perfect number is greater than $10^{1500}$.

Use factor chains to forbid the factors in $S=\{127,19,7$, $11,331,31,97,61,13,398581,1093,3,5,307,17\}$, in this order. These chains are constructed using branchings. To branch on a prime $\mathrm{p}$ means that we sequentially branch on all possible components pa. To branch on a component pa for $p$ prime means that we suppose pa $\mathrm{kN}$, and thus pa $\times$ _(pa) $2 \mathrm{~N}$ since $\operatorname{gcd}(\mathrm{pa}, \quad(\mathrm{pa}))=1$. Then, if we do not reach a contradiction at this point, we recursively branch on a prime factor of $\mathrm{N}$ that has not yet been branched on. If there is no known other factor of $\mathrm{N}$. In this section, we branch on the overall largest available prime factor and use the following contradictions:

(1) The abundancy of the current number is strictly greater than 2 .

(2) The current number is greater than 101500 .

When branching on a prime $p$, we have to consider various cases depending on the multiplicity of $\mathrm{p}$ in $\mathrm{N}$. We stop when the multiplicity a of $\mathrm{p}$ is such that $\mathrm{pa}>101500$, we consider only the multiplicities such that $a+1$ is prime. This is because _ (pa) $\left.\right|_{-} \mathrm{p}(\mathrm{a}+1) \mathrm{t}-1_{-}$, so any contradiction obtained thanks to the factors of _ (pa) when supposing pa $\mathrm{k} \mathrm{N}$ also gives a contradiction in the case $p(a+1) t-1 k N$. So pa is a representative for all $\mathrm{p}(\mathrm{a}+1) \mathrm{t}-1$, and to compute lower bounds on the abundancy or the size to test for contradictions, suppose that the multiplicity of $\mathrm{p}$ is exactly a.

\subsection{Perfect Numbers and Prime Divisors}

In number theory, the prime factors or divisors of a positive integer are the prime numbers that divide that integer exactly. Mathematicians discover this useful for finding an Odd Perfect Number.

Theorem 4.2 by Ochem and Rao: The total number of prime factors of an odd perfect number is at least 101 .

\subsection{Largest Prime Factor}

According to Ben Stevens on his paper entitled "A Study on Necessary Conditions for Odd Perfect Numbers", Goto upon using an algorithm, show that $10^{8}$ is an appropriate lower bound for the largest prime factor of an odd perfect number. Lannucci on the other hand, shows that the second large stand third largest prime divisors must exceed 10000 and 100 respectively. [13]

Theorem 4.3 by Goto (2008): The largest component of an odd perfect number is greater than 108 .

We use the same algorithm as in the previous section to forbid every prime less than 108 using the following contradictions:

(1) The abundancy of the current number is strictly greater than 2 .

(2) The current number has a component greater than 1062

To quickly reach a large component, we branch on the largest available prime. There is no unfactored composite here, and thus, no roadblock, since every number is less than 1062 and thus has been easily factored. Suppose now that N is an odd perfect number with no prime factor less than 108 and no component pe $>1062$. First, the exponent e of any prime factor pis less than 8 , since otherwise pe $>(108) 8>$ 1062. The exponent of the special prime $\mathrm{p} 1$ is thus 1 , because 3 |_(p5) and $3 \nmid N$. So $N$ has a prime de

Composition $\mathrm{N}=\mathrm{p} 1 \mathrm{Q} 2 \mathrm{i}=1$ p2i,2Qn4i=1 p4i,4Qn6i=1 p6i,6.

Let_ $(\mathrm{x})$ denote the number of primes less than or equal to $\mathrm{x}$. In the following, These known values of $(\mathrm{x})$ [8]:

(1) $(108)=5761455$

(2) $(3 \times 1010)=1300005926$

(3) $(32 \times 1014)=92295556538011$

(4) $(98 \times 1014)=273808176380030$

It is well-known that for primes $\mathrm{q}, \mathrm{r}$, and s such that $\mathrm{q} \mid$ $\left(\mathrm{rs}^{-1}\right)$, either $\mathrm{q}=\mathrm{s}$ or $\mathrm{q} \equiv 1 \bmod \mathrm{s}$. So if $\mathrm{pj}, \mathrm{e}^{\prime} \mid$ (pei, e), then $\mathrm{pj}, \mathrm{e}^{\prime} \equiv 1 \bmod (\mathrm{e}+1), \operatorname{since}(\mathrm{e}+1) \nmid \mathrm{N}$. We thus have $\mathrm{e}^{\prime}$ $6=\mathrm{e}$, since otherwise e +1 would divide _(pej, e) (that is, _ $\left(p^{\prime} j \mathrm{j}, \mathrm{e}^{\prime}\right)$ ), but not N. Moreover, _(pei, e) cannot be prime unless it is the special prime p1. Suppose to the contrary that (pei, e) $=p j, e^{\prime}$. Then pe'j, e $e^{\prime}$ is a componentof N. Since $e^{\prime}$ $\overline{6}=\mathrm{e}$, we have that $\mathrm{ee}^{\prime} \geq 8$, so that $\mathrm{pe}^{\prime} \mathrm{j}, \mathrm{e}^{\prime}=\left(\right.$ (pei, e)) $\mathrm{e}^{\prime}>$ (pei, e) $\mathrm{e}^{\prime}=($ pi, e)ee $>(108) 8>1062$. So each _(pei, e) produces at least two factors or the special prime.

Let $\mathrm{n} 2,2$ be the number of primes pi, 2 such that_(p2, 2) = $\mathrm{q} \times \mathrm{r}$ where $\mathrm{q}<\mathrm{r}$, qand $\mathrm{r}$ primes. Let $\mathrm{n} 2,3$ be the number of primes pi,2 such that_( $22 \mathrm{i}, 2)$ factors into at least three not necessarily distinct primes.

By the above, we have (1) $\mathrm{n} 2 \leq \mathrm{n} 2,2+\mathrm{n} 2,3+1$. By counting the number of primes produced by the factors _ (p2i, 2), we obtain(2 ) 2n2,2 + 3n2,3 $\leq 4 \mathrm{n} 4+6 \mathrm{n} 6+1$. For e $\in\{4,6\}$, we have pi, e $<32 \times 1014$, since otherwise pei, e $>(32 \times 1014) 4>1062$. Suppose that a prime pi, 2 is such that $(\mathrm{p} 2 \mathrm{i}, 2)=\mathrm{q} \times \mathrm{r}$ where $\mathrm{q}<\mathrm{r}, \mathrm{q}$ and $\mathrm{r}$ primes. Then $r>p i, 2$, and by previous discussion, either $r=p 1$ or $\mathrm{r}=\mathrm{pi}^{\prime}$, efor $\mathrm{e} \in\{4,6\}$ This implies that at least (n2, $2-$ 1) primes pi, 2 are smaller than the largest prime pi, e for e

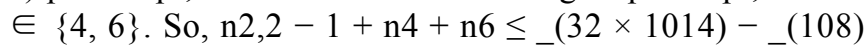
$=92295550776556$ which gives(3) n2,2 + n4 + n6 $\leq$ 92295550776557. Similarly, pi, $6<3 \times 1010$ since otherwise p6i, $6>1062$. So, n6 $\leq$ _ $(3 \times 1010)-$ _ $(108)$, which gives $n 6 \leq 1294244471$ Now, we consider an upper bound on the abundancy of primes greater than 108. Use equation $\mathrm{Yp}<\mathrm{xp}$ prime $\mathrm{p}-1<\mathrm{e} \ln (\mathrm{x}) \_1+12 \ln 2(\mathrm{x})$ _where $=0.5772156649 \ldots$ is Euler's constant. 
Compute that $\mathrm{Y} p<108 \mathrm{p}$ prime $\mathrm{p}-1>\mathrm{c} 1=$ 32.80869860873870116 and we obtain $\mathrm{Y} \quad 108<\mathrm{p}<98 \times 1014 \mathrm{p}$ prime $\mathrm{p}-1<\mathrm{e} \ln (98 \times 1014) \_1+12 \ln 2(98 \times 1014) \_/ \mathrm{c} 1<2$. By the above, we have $1+\mathrm{n} 2+\mathrm{n} 4+\mathrm{n} 6>(98 \times 1014)-(108)=$ 273808170618575 , which gives,(5) $27 \overline{3} 808170618575 \leq \mathrm{n} 2$ $+\mathrm{n} 4+\mathrm{n} 6$. The combination $3 \times(1)+1 \times(2)+7 \times(3)+2 \times$ $(4)+3 \times(5)$ gives $6 \mathrm{n} 2,2+175353067930880 \leq 0$, a contradiction

\subsection{On Odd Near-Perfect and Deficient - Perfect Numbers}

Positive integer $\mathrm{n}$ a near-perfect number, if it is sum of all its proper divisors, except one of them. An Odd Near-Perfect Number is a number that is odd and near perfect at the same time. Let ó(n) denote the sum of the divisors of $n$. We say that $\mathrm{n}$ is a Deficient-Perfect Number if ó(n) $<2 \mathrm{n}$. [14]

Theorem 3.4.1 BY Adajar, $C$.

I There are finitely many odd near-perfect integers with exactly $\mathrm{k}$ prime divisors.

II There are finitely many odd deficient -perfect integers with exactly $\mathrm{k}$ prime divisors.

Lemma 3.4.2 BY Adajar, $C$.

Let $\alpha$ be a rational number. Let $\mathrm{N}$ be an odd number with at most $\mathrm{k}$ prime factors, and suppose that ó $(\mathrm{N})=\alpha \mathrm{N}$ then $\mathrm{N}<(\mathrm{m}=1)^{\wedge} 4^{\wedge} \mathrm{k}$. where $\mathrm{m}$ is the denominator of $\alpha$ as expressed in lowest term.

Lemma 3.4.1 BY Adajar, $C$.

Suppose $\mathrm{p} 1, \mathrm{p} 2, \ldots, \mathrm{pk}$ are $\mathrm{k}$ distinct primes, and M€R

I The set $\left\{o^{\prime}-1\left(\mathrm{p} 1^{\wedge} \mathrm{r} 1 \mathrm{p} 2^{\wedge} \mathrm{r} 2 \ldots \mathrm{pk}^{\wedge} \mathrm{rk}\right) \geq \mathrm{M}: \mathrm{r} 1, \mathrm{r} 2, \ldots \mathrm{rk} € 2 \mathrm{~N}\right.$ if nonempty, contains its minimum

II The set $\left\{o^{\prime}-1\left(\mathrm{p} 1^{\wedge} \mathrm{r} 1 \mathrm{p} 2^{\wedge} \mathrm{r} 2 \ldots \mathrm{pk}{ }^{\wedge} \mathrm{rk}\right) \leq \mathrm{M}: \mathrm{r} 1, \mathrm{r} 2, \ldots \mathrm{rk} € 2 \mathrm{~N}\right.$ $\mathrm{U}\{\infty\}\}$ if nonempty, contains its minimum

\subsection{Necessary Conditions for Odd Perfect Numbers}

A perfect number is a natural number (an integer greater than zero), whose sigma function equals twice thenumber itself, i.e. $\sigma(\mathrm{N})=2 \mathrm{~N}$. The sigma function is the sum of all divisors. As an example, 6 is a perfect number. This is due to $\sigma(6)=12$; this is because $1+2+3+6=12$. The existence of even $\mathrm{p}$ : erfect numbers is easily documented, as shown above. Euclid proved that there is a one-to-one correspondence between even perfect numbers and Mersenne primes (a prime being of the form $2 p-1$, where $p$ is also prime). An even perfect number will always be of the form (2p-1) $2 p-1$. The proof is as follows. [15]

Theorem 3.5.1 by Stevens, Ben

Let $\mathrm{n}$ be a positive integer. Then, $\mathrm{n}$ is an even perfect numberiff $n=2 p-1(2 p-1)$ where $2 p-1$ isprime (which implies $\mathrm{p}$ is prime, by Mersenne).

\section{Conclusion}

The following conclusions were derived from the outcome of the study:

(1) Lower Bound for Odd Perfect Numbers has a lower bound of $10^{1500}$. This is the latest known information on the lower bound of odd perfect numbers. -
(2) Perfect numbers and prime divisors according to Ben Stevens on his paper entitled "A Study on Necessary Conditions for Odd Perfect Numbers", studies have shown that in order for an odd perfect number to exist, there has to be a minimum number of prime divisors. The total number of prime factors/divisors of an odd perfect number is at least 101 .

(3) Largest Prime Factor is any of the prime numbers that can be multiplied to give the original number. Mathematicians discovered that they can use this in studying Odd Perfect Numbers. Goto upon using an algorithm, show that $10^{8}$ is an appropriate lower bound for the largest prime factor of an odd perfect number. Lannucci on the other hand, shows that the second large stand third largest prime divisors must exceed 10000 and 100 respectively. -

(4) On Odd Near-Perfect and Deficient- Perfect Numbers The existence of an odd perfect number remains a big mystery in the field of number theory. The existence of an odd perfect numbers is important due to the fact that it will help to solve some unsolved math conjecture.

(5) Using the four inputs used, it was proved that the existence of an odd perfect number is possible, provided that the necessary conditions are met.

\section{References}

[1] Perfect number. (2018). Retrieved from https://en.wikipedia.org/wiki/Perfect_number.

[2] Perfect Numbers. Retrieved from https://math.tutorvista.com/number-system/perfectnumbers.html.

[3] Bogomolny, Alexander. (2017) Pythagorean Triples and Perfect Numbers.

[4] Mersenne Prime. (2018). Retrieved from https://en.wikipedia.org/wiki/Mersenne_prime.

[5] Caldwell, Chris K. (2016). Mersenne Primes: History, Theorems and Lists. Retrieved from https://primes.utm.edu/mersenne/.

[6] Brown, D. R. (2011). "Odd Perfect Numbers".

[7] Carr, Aver. (2013). "Odd Perfect Numbers: Do They Exist?"

[8] The Largest Perfect Number (2016, February 7). Retrieved from http://www.mathnasium.com/www-mathnasium-comfremont-news-the-largest-erfect-number.

[9] Review Papers. (2001). Retrieved from http://websites.uwlax.edu/biology/ReviewPapers.html.

[10] Bhat, Adi. (2018).

http://libguides.usc.edu/writingguide/qualitative.

[11] What is the difference between a research paper and review paper. (2015). Retrieved from https://www.editage.com/insights/what-is-the-differencebetween-a-research-paper-and-a-review-paper. 
[12] Sarmiento, Janilo. (2012). Retrieved from https://www.slideshare.net/janilosarmiento/the-ipo-model-ofevaluation-input, process, output.

[13] Stevens, Ben. (2012). A Study on Necessary Conditions for Odd Perfect Numbers".
[14] Adajar, Carlo. (2016, University of the Philippines Diliman), On Odd Near-Perfect and Deficient- Perfect Numbers.

[15] Almost Perfect Number. (2018). Retrieved from https://en.wikipedia.org/wiki/Almost_perfect_number. 